\title{
The social brain network in $22 q 11.2$ deletion syndrome: a diffusion tensor imaging study
} (a) CrossMark

\author{
Amy K. Olszewski ${ }^{1}$, Zora Kikinis², Christie S. Gonzalez ${ }^{3}$, loana L. Coman ${ }^{1}$, Nikolaos Makris ${ }^{2,4}$, Xue Gong ${ }^{2}$, \\ Yogesh Rathi ${ }^{2}$, Anni Zhu ${ }^{2}$, Kevin M. Antshel ${ }^{3}$, Wanda Fremont ${ }^{1}$, Marek R. Kubicki ${ }^{2,5}$, Sylvain Bouix ${ }^{2}$, \\ Martha E. Shenton ${ }^{5,6}$ and Wendy R. Kates ${ }^{1 *}$
}

\begin{abstract}
Background: Chromosome 22q11.2 deletion syndrome (22q11.2DS) is a neurogenetic disorder that is associated with a 25-fold increase in schizophrenia. Both individuals with 22q11.2DS and those with schizophrenia present with social cognitive deficits, which are putatively subserved by a network of brain regions that are involved in the processing of social cognitive information. This study used two-tensor tractography to examine the white matter tracts believed to underlie the social brain network in a group of 57 young adults with 22q11.2DS compared to 30 unaffected controls.

Results: Results indicated that relative to controls, participants with 22q11.2DS showed significant differences in several DTI metrics within the inferior fronto-occipital fasciculus, cingulum bundle, thalamo-frontal tract, and inferior longitudinal fasciculus. In addition, participants with 22q11.2DS showed significant differences in scores on measures of social cognition, including the Social Responsiveness Scale and Trait Emotional Intelligence Questionnaire. Further analyses among individuals with 22q11.2DS demonstrated an association between DTI metrics and positive and negative symptoms of psychosis, as well as differentiation between individuals with 22q11.2DS and overt psychosis, relative to those with positive prodromal symptoms or no psychosis.
\end{abstract}

Conclusions: Findings suggest that white matter disruption, specifically disrupted axonal coherence in the right inferior fronto-occipital fasciculus, may be a biomarker for social cognitive difficulties and psychosis in individuals with 22q11.2DS.

Keywords: 22q11.2 deletion syndrome, Social brain network, Social cognition, Two-tensor tractography, White matter tracts

\section{Background}

Chromosome 22q11.2 deletion syndrome (22q11.2DS), also known as velo-cardio-facial syndrome (VCFS), or DiGeorge syndrome, is a genetic neurodevelopmental disorder that occurs as a result of an interstitial deletion of 40-50 genes on the long arm of chromosome 22 [1]. The most recent estimate of the syndrome's incidence is 1:992 live births [2]. The deletion is associated

\footnotetext{
*Correspondence: katesw@upstate.edu

1 Department of Psychiatry, SUNY Upstate Medical University, 750 E. Adams St., Syracuse, NY 13210, USA

Full list of author information is available at the end of the article
}

with a myriad of physical features, including distinctive facial characteristics, palatal abnormalities, and cardiac anomalies [3]. In addition to these physical characteristics, individuals with 22q11.2DS often possess a distinct neuropsychological profile, consisting of a full scale IQ in the borderline range, as well as deficits in executive function, working memory, and visuospatial abilities [4-6]. 22q11.2DS is also associated with multiple psychiatric comorbidities, including mood disorders [7-10], anxiety disorders $[3,9,11-13]$, attention-deficit/hyperactivity disorder (ADHD; [9-11, 13]), autism spectrum disorder (ASD; $[5,14,15]$ ), and, in up to $30-40 \%$ of adults, 
psychotic disorders such as schizophrenia [16, 17]. In fact, aside from having a monozygotic twin with schizophrenia, 22q11.2DS is the next highest risk for developing schizophrenia [16].

\section{Social processing and the social brain}

Many individuals with 22q11.2DS also experience social difficulties, including shyness, withdrawal, social immaturity, and deficits in social cognition [18-21]. Social cognition refers to the mental processes that subserve social interactions [22] and includes theory of mind, i.e., being able to see things from another's perspective, also referred to as "mentalizing skills," attributional style, social perception, and emotional processing abilities [22, 23]. Importantly, social cognitive impairments have also been identified in individuals with schizophrenia [24] and autism spectrum disorders [25].

Of further note, functional brain imaging studies have identified two major social networks that are involved in social cognition tasks: a mirror network, which is involved in reading another individual's body language, and a mentalizing network that allows for social mentalizing, or taking another's perspective [26-28]. These networks involve several brain regions in the prefrontal (i.e., dorsolateral-ventrolateral-medial prefrontal and premotor cortices), pregenual and dorsal anterior cingulate regions, the insula, the amygdala, inferior parietal lobule and precuneus as well as the temporopolar and the temporo-parieto-occipital junction areas [24, 29, 30]. Insofar as studies have shown associations between aberrant connectivity within the mentalizing network and social cognitive difficulties among individuals with schizophrenia [31-34], ASD [35-38], and 22q11.2DS [39, 40], our primary interest was to examine the white matter tracts underlying social cognition in 22q11.2DS.

\section{Structural imaging: diffusion tensor imaging}

Recent advances in imaging, including diffusion tensor imaging (DTI) and fiber tractography post-processing analyses, have enabled the use of noninvasive methods to measure structural white matter tract integrity in vivo by examining the diffusion of water molecules in the brain. DTI metrics, including fractional anisotropy (FA), a measure of white matter integrity, radial diffusivity (RD), a purported measure of myelin integrity, and axial diffusivity (AD), a purported measure of axonal integrity, make it possible to quantify differences in these metrics across groups. Studies in individuals with 22q11.2DS show altered white matter microstructure in long-range and limbic connections (see review by [41]), including fronto-parietal, fronto-temporal, and parieto-occipital networks, as well as in cingulum bundle $(\mathrm{CB})$, anterior limb of the internal capsule (ALIC), anterior thalamic radiation and uncinate fasciculus (UF), all areas known to show abnormalities in individuals with schizophrenia [41-46].

\section{DTI tracts related to social functioning}

Several white matter tracts are believed to play important roles in the transmission of social information. For example, the $C B$ is an associative bundle of fibers running through the cingulate gyrus around the corpus callosum. Longer fibers run from the anterior temporal gyrus to the orbitofrontal cortex, while shorter fibers connect the four lobar regions of the brain and the cingulate cortex [47]. The location of the $\mathrm{CB}$ within the limbic system suggests that it plays an important role in emotional information processing. The UF is also part of the limbic system, and connects the amygdala to the anterior temporal lobe and orbitofrontal cortex [47]. Thus, the UF is also believed to play a role in emotion processing.

The superior longitudinal fasciculus (SLF) is composed of four separate components, SLF I-SLF III and the arcuate fasciculus. The SLF II composes the central core of white matter above the insula, and is believed to be the major link between the parietal lobe and prefrontal cortex, thereby implicating a role in the perception of visual space [48]. The inferior fronto-occipital fasciculus (IFOF) connects the ventral occipital lobe to the orbitofrontal cortex, and is involved in visual processing/facial emotion recognition [47]. A study by DeRosse and colleagues [49] found that lower FA in the IFOF is related to higher levels of schizotypy, indicating a possible role for IFOF in experience/affect sharing [49]. The inferior longitudinal fasciculus (ILF) connects the occipital and temporal lobes. While not much is known regarding the ILF's functionality, it is believed to be involved in face recognition and visual object perception $[47,50,51]$. Finally, the anterior thalamic radiation (ATR) connects the anterior nuclear and midline nuclear groups of the thalamus with the frontal lobe [52]. The ATR is located in the thalamolimbic area, and hypoconnectivity of the ATR has been demonstrated in males with ASD, a disorder associated with social cognitive impairment [53]. Studies have also shown that functional connectivity (i.e., fMRI) reflects structural connectivity (i.e., DTI) in the mentalizing network $[54,55]$.

The aim of the current project was to explore structural DTI tracts hypothesized to be involved in the social brain, as well as to investigate associations with measures of social and emotional processing. We predicted that there would be significant differences between individuals with 22q11.2DS and controls in DTI metrics, including $\mathrm{FA}, \mathrm{RD}, \mathrm{AD}$, and the number of streamlines (an estimate of fiber bundles). Following the expected reductions in DTI metrics, we also hypothesized: (1) FA 
in IFOF, SLF, the thalamo-frontal tract, and CB would be correlated with a measure of social responsiveness; (2) FA in ILF and IFOF would be associated with a measure of experience and affect sharing; and (3) FA in UF and IFOF would be associated with emotion regulation and cognitive reappraisal. Finally, we predicted that alterations in DTI metrics in 22q11.2DS would be associated with a dimensional measure of both positive and negative symptoms of prodromal/overt psychosis.

\section{Methods}

\section{Participants}

The imaging and psychiatric data presented in this study were derived from a subsample of participants enrolled in a longitudinal study of risk factors for psychosis in 22q11.2DS [56]. This subsample consists of 57 participants with 22q11.2DS, 12 unaffected siblings, and 18 community controls who returned for the fourth time point of the study. Participants were recruited from the International Center for Evaluation, Treatment, and Study of Velo-Cardio-Facial Syndrome at SUNY Upstate Medical University, parent support groups, and the surrounding community. Presence of the 22q11.2 deletion was confirmed with fluorescence in situ hybridization (FISH). Informed consent was obtained under protocols approved by the medical center's institutional review board. Initial statistical analyses comparing sibling and community controls did not differ for any of the measures utilized in this study (see Additional file 1). Therefore, we combined the sibling and community controls into one control group for the remainder of the analyses. Demographic information is provided in Table 1.

Several papers examining white matter microstructure have been published on this cohort based on assessment at the third time point of the study (when they were between the ages of 15 and 21) [43, 45, 46]. However, this is the first paper to examine white matter microstructure based on participants' assessments at the 4th timepoint of the study, when they were between the ages of 18 and 24 years, representing the age window at which this cohort is at highest risk for developing psychotic symptoms. Moreover, whereas previous papers have been based on imaging data acquired from a 1.5 Tesla scanner, the current study is based on data acquired from a 3 Tesla scanner, utilizing state-of-the-art two-tensor tractography to measure white matter microstructure.

Exclusion criteria for all participants included: presence of a seizure disorder, fetal exposure to alcohol or drugs, parent-reported elevated lead levels, birthweight under $2500 \mathrm{~g}$, history of loss of consciousness lasting longer than $15 \mathrm{~min}$, paramagnetic implants, or orthodontic braces. Potential community control participants were also excluded if there was a personal or family history of
Table 1 Participant demographics

\begin{tabular}{lll}
\hline Demographic variable & 22q11.2DS (N = 57) & Control (N= 30) \\
\hline Age [Mean (SD)] & $20.87(2.29)$ & $20.97(1.46)$ \\
Gender [N (\%)] & & \\
Male & $31(54.4)$ & $17(56.7)$ \\
Female & $26(45.6)$ & $13(43.3)$ \\
FSIQ [Mean (SD)] & $74.54(11.82)^{* * *}$ & $109.47(16.02)$ \\
Race [N (\%)] & & \\
Native American & 0 & $1(3.3)$ \\
Asian & $1(1.8)$ & $2(6.7)$ \\
African American & 0 & $1(3.3)$ \\
Caucasian & $51(89.5)$ & $24(80.0)$ \\
More than one & $2(3.5)$ & $1(3.3)$ \\
Unknown & $3(5.3)$ & $1(3.3)$ \\
Psychiatric diagnosis [N (\%)] & & $1(3.3)$ \\
Mood disorder & $7(12.3)$ & $5(16.7)$ \\
Anxiety disorder & $16(28.1)$ & 0 \\
ASD & $7(12.3)$ & $5(16.7)$ \\
ADHD & $9(15.8)$ & 0 \\
Psychotic disorder & $4(7.0)$ & 0 \\
Other & $3(5.2)$ & $7(23.3)$ \\
Any psychiatric diagnosis & $27(47.4)$ & \\
\hline
\end{tabular}

22q11.2DS, 22q11.2 deletion syndrome; FSIQ, full scale IQ; ASD, autism spectrum disorder; ADHD, attention-deficit/hyperactivity disorder

${ }^{* * *} p<.0001$

schizophrenia or bipolar disorder [57]. All participants were screened for psychiatric disorders using the structured clinical interview for DSM-IV-TR (SCID).

\section{Procedures}

As described in previous studies $[7,11,14,58]$, each participant and parent/caregiver completed measures of cognitive and/or social, emotional, and behavioral functioning. All diagnostic interviews were completed by a licensed psychiatrist or psychologist, and all neuropsychological measures were administered by an experienced doctoral-level examiner. Due to the facial features characteristic of 22q11.2DS, evaluator blindness to group assignment was not possible. A licensed psychologist or trained student assistant familiar with the measures double scored all protocols to ensure scoring accuracy. Caregivers completed behavior rating scales and background information while the children and adolescents were completing neuropsychological measures.

\section{Measures}

Wechsler Adult Intelligence Scale, third edition (WAIS-III) The WAIS-III [59] is a test of cognitive ability that provides intelligence quotient (IQ) scores, including a full scale IQ (FSIQ), verbal IQ (VIQ), and performance 
IQ (PIQ) for individuals 16 years of age and older. The WAIS-III consists of several subtests (mean $=10$, $\mathrm{SD}=3$ ), which measure various domains. The WAIS-III has outstanding reliability, with internal consistency reliability coefficients at or above .93 for the WAIS-III FSIQ, VIQ, and PIQ [60].

\section{Social Responsiveness Scale (SRS)}

The SRS is a 65-item parent report questionnaire designed to assess the different dimensions of interpersonal behavior, communication, and repetitive/stereotypic behaviors that are characteristic of autism spectrum disorders [61]. Psychometric properties of the SRS are excellent, with total score alpha coefficients above .90 for both males and females in both clinical and normative sample [61]. In this study, we used the adult research version of the SRS to assess for social deficits, with higher total raw scores indicative of more severe social impairment [62].

\section{Junior Schizotypy Scale (JSS)}

The JSS is a 50-item self-report questionnaire used to measure schizotypal personality traits in adolescents, which are believed to indicate a predisposition to schizophrenia in adulthood. The JSS provides scores for five subscales, each of which reflect a particular aspect of schizotypy: cognitive, perceptual, social, impulsive nonconformity, and physical anhedonia [63]. Higher scores on each scale indicate higher levels of schizotypy.

\section{Trait Emotional Intelligence Questionnaire (TEIQue)}

The TEIQue [64] is a 153-item questionnaire based on trait emotion intelligence theory. The TEIQue uses a 7-point Likert response scale (1: disagree completely; 7: agree completely) to measure the level of various facets of emotional intelligence. Higher scores on each facet represent better levels of perceived abilities and dispositions [64]. This study used the parent version of the TEIQue, in which the parent or caregiver rated the participant's emotional intelligence. For the current study, we were particularly interested in the four facets that relate to social cognition; therefore, our analyses focused on emotion regulation (the degree to which an individual has control over his or her emotions), empathy (the ability to take another's perspective), social awareness (social skills and the ability to adapt to and interact in various social situations), and emotion perception (the ability to perceive one's own and others' emotions).

\section{Structured interview for prodromal syndromes (SIPS)}

The SIPS [65] is a scale that measures the severity and change of individuals who are experiencing pre-psychotic symptoms. The SIPS consists of five positive symptom items, six negative symptom items, four items related to disorganized symptoms, and four general symptom items, each of which are rated on a severity scale ranging from 0 (never or absent) to 6 (severe and psychotic, or extreme). The SIPS was administered to all study participants and separately to their parents. The positive symptoms (SIPS PS) and negative symptoms (SIPS NS) scores were used for the purpose of determining prodromal/ psychotic symptoms.

\section{MRI acquisition/DTI processing \\ Scan acquisitions}

For the time point examined in this study (Time Four), images were acquired using a 3T Siemens Magnetom Tim Trio scanner (Siemens Medical Solutions, Erlangen, Germany).The high resolution anatomic scan consisted of an ultrafast gradient echo 3D sequence (MPRAGE) with PAT k-space-based algorithm GRAPPA. The parameters included: echo time $=3.31 \mathrm{~ms}$; repetition time $=2530 \mathrm{~ms}$; matrix size $=256 \times 256$; field of view $(\mathrm{FOV})=256 \mathrm{~mm}$; slice thickness $=1 \mathrm{~mm}$. The DWI sequence consisted of 64 transverse slices with no gaps and $2.0 \mathrm{~mm}$ nominal isotropic resolution $(\mathrm{TR} / \mathrm{TE}=8600 / 93 \mathrm{~ms}$, FOV $=244 \times 244$, data matrix $=96 \times 96$, zero-filled and reconstructed to $256 \times 256$ ). Diffusion weighting was applied along 64 directions with a $\boldsymbol{b}$ factor $=700 \mathrm{~s} / \mathrm{mm}^{2}$. One minimally weighted volume $\left(b_{0}\right)$ was acquired within each DWI dataset. The total scan time to acquire the DWI dataset was 4 min., $52 \mathrm{~s}$. A high resolution T2 scan was also obtained to align with the DWI images.

\section{Diffusion tensor imaging preprocessing}

An in-house script was used to correct for eddy current distortions and head motion. This script registered each diffusion-weighted volume to the baseline volume using FSL (http://fsl.fmrib.ox.ac.uk) linear registration software "FLIRT". Motion correction was not performed.

\section{Whole brain tractography}

For the purposes of this study, we used two-tensor tractography to determine white matter tracts/bundles. As compared to single tensor tractography, two-tensor tractography offers a better fiber representation in both fiber branching and fiber crossing by computing two tensors for each voxel [66]. We generated fiber tracts from DWI images using the Unscented Kalman Filter (UKF) based on two-tensor tractography algorithm [67]. Tract seeding was completed in every voxel where the primary single tensor FA value was larger than .18, with each voxel seeded 10 times. Fibers between neighboring voxels were traced following the direction of the primary tensor component. Fibers were terminated when the primary tensor FA value was less than 15 . 


\section{FreeSurfer parcellations and registration to DTI space}

We used FreeSurfer software (http://surfer.nmr.mgh.harvard.edu) to obtain regions of interest via an automated approach, which parcellated the cortical and white matter regions. We applied FreeSurfer software to segment T1-weighted SPGR images into 34 bilateral, cortical and white matter regions for each participant [68]. The label map with FreeSurfer-generated regions of interest was registered to the DWI space by first diffeomorphically registering a T2 image in the same space as the SPGR image to the baseline DWI image of the same participant using the FLIRT algorithm of the FSL software [69], and then applying this diffeomorphism to register the FreeSurfer-generated label map to the DWI space for the same participant. We performed this transformation of the FreeSurfer label map to DWI space for each participant.

\section{White matter query language}

White matter query language (WMQL) was used to extract fiber tracts from the two-tensor whole brain tractography [70]. WMQL was designed to use neuroanatomical definitions of white matter to estimate fiber tracts [47]. Fiber tract definitions were based on cortical regions known to be connected via these fiber bundles, as well as on white matter regions where the fiber tract is expected to project. These definitions used the FreeSurfer-generated parcellations of cortical and white matter regions [70]. We implemented WMQL queries to extract the left and right hemisphere $\mathrm{CB}$, ILF, SLF II, and thalamo-frontal tracts. Because the WMQL approach does not rely on a specific atlas, label maps other than those generated by FreeSurfer can also be used. We extracted the right and left hemisphere UF and IFOF from the 2-tensor whole brain tractography using a label map with manually drawn ROIs. The DTI metrics of $F A, A D$, and $R D$ were extracted from the entire fiber tract and the mean values were computed. WMQL also allowed us to calculate the number of streamlines for each tract, and only tracts with more than 10 streamlines were reported. Two-tensor tractography was first used in a study on first-episode schizophrenia [71], and in later studies combining two-tensor tractography with WMQL queries $[48,70]$. It has not, until this study, been used in 22q11.2DS.

\section{Statistical analyses}

The data were examined for normality in order to ensure they met criteria for the assumptions of statistical tests to be used. For variables that did not meet the assumption of normality (i.e., skewness and/or kurtosis $<1.0$ ), we applied a $\log$ transformation to normalize the data. For data that remained nonnormal after transformation, we created standardized residuals so that nonparametric tests could be used. Where appropriate, analysis of covariance (ANCOVA) and multivariate analyses of covariance (MANCOVAs), using either hemispheric white matter volume or FSIQ as covariates, were used to investigate possible differences between groups on DTI metrics and social brain measures, respectively. Follow up ANOVAs examined which dependent variables drove the significant differences in DTI metrics and scores on social brain measures. In the Results section, we report on DTI metrics that passed Bonferroni correction for multiple comparisons on each hemispheric tract, as well as subscales of social brain measures that passed Bonferroni correction for each measure. Individual Bonferroni correction thresholds varied according to the number of dependent variables within each measure. We also used Pearson correlations to examine relationships between significantly different DTI tract metrics and social brain measures. We used Spearman correlations for variables that remained nonnormal after log transformation. Finally, we used zero-inflated Poisson (ZIP) regressions to analyze the associations between social measures and positive prodromal symptoms, and DTI tracts and positive prodromal symptoms. All data were analyzed using SPSS v. 23 or Stata v. 12.0.

\section{Results}

\section{DTI tract differences}

To explore group differences in DTI metrics, we conducted a multivariate analysis of covariance (MANCOVA), using hemispheric white matter volume as a covariate, to compare participants with 22q11.2DS and controls. Table 2 presents the descriptive statistics for all DTI metrics, and Table 3 includes results of the MANCOVA. Compared to controls, participants with 22q11.2DS showed a significant increase in left FA, suggesting increased white matter integrity, and a significant decrease in left RD (suggesting decreased white matter integrity) within the IFOF; a significant increase in right hemisphere FA (increased white matter integrity) and a significant decrease in right RD (decreased white matter integrity) within the $\mathrm{CB}$; a significant increase in right hemisphere FA (increased white matter integrity) and a significant decrease in right hemisphere RD (decreased white matter integrity) within the thalamo-frontal tract; and a significant decrease in right hemisphere RD for the ILF. Results for the SLF did not pass the Bonferroni correction.

Nonparametric Mann-Whitney $U$ tests were conducted for the non-normally distributed right hemisphere IFOF RD $(p<.0001)$ and FA $(p=.001)$, as well as the right hemisphere UF number of streamlines $(p=.010)$, indicating group differences in these metrics. 
Table 2 Descriptive statistics for DTI metrics

\begin{tabular}{|c|c|c|c|c|}
\hline & \multicolumn{2}{|c|}{$22 q 11.2 D S$} & \multicolumn{2}{|l|}{ Control } \\
\hline & $M$ & $S D$ & $M$ & $S D$ \\
\hline \multicolumn{5}{|l|}{ Left hemisphere } \\
\hline UF FA & .589 & .036 & .591 & .027 \\
\hline UF RD & .000467 & .0000424 & .000471 & .0000328 \\
\hline UF AD & .00136 & .0000365 & .00138 & .0000342 \\
\hline $\begin{array}{l}\text { UF number of stream- } \\
\text { lines }\end{array}$ & 129.179 & 119.85 & 152.774 & 100.28 \\
\hline IFOF FA & .691 & .025 & .662 & .029 \\
\hline IFOF RD & .000373 & .0000282 & .000414 & .0000352 \\
\hline IFOF AD & .00147 & .0000373 & .00148 & .0000292 \\
\hline $\begin{array}{l}\text { IFOF number of } \\
\text { streamlines }\end{array}$ & 336.927 & 242.56 & 421.000 & 187.77 \\
\hline CB FA & .608 & .032 & .599 & .032 \\
\hline CB RD & .000429 & .0000338 & .000448 & .0000349 \\
\hline$C B A D$ & .00132 & .0000355 & .00135 & .0000350 \\
\hline $\begin{array}{l}\text { CB number of stream- } \\
\text { lines }\end{array}$ & 1228.754 & 398.591 & 1119.677 & 344.107 \\
\hline ILF FA & .645 & .039 & .640 & .047 \\
\hline ILF RD & .000423 & .0000535 & .000430 & .0000470 \\
\hline ILF AD & .00143 & .0000514 & .00146 & .0000719 \\
\hline $\begin{array}{l}\text { ILF number of stream- } \\
\text { lines }\end{array}$ & 20.158 & 18.817 & 11.000 & 9.501 \\
\hline SLFFA & .643 & .034 & .639 & .041 \\
\hline SLF RD & .000418 & .0000498 & .000420 & .0000482 \\
\hline SLF AD & .00141 & .0000496 & .00141 & .0000519 \\
\hline $\begin{array}{l}\text { SLF number of } \\
\text { streamlines }\end{array}$ & 66.774 & 55.734 & 108.355 & 79.342 \\
\hline Thalamo-frontal FA & .629 & .022 & .624 & .016 \\
\hline Thalamo-frontal RD & .000407 & .0000218 & .000447 & .0000182 \\
\hline Thalamo-frontal AD & .00132 & .0000288 & .00134 & .0000223 \\
\hline $\begin{array}{l}\text { Thalamo-frontal } \\
\text { number of } \\
\text { streamlines }\end{array}$ & 760.772 & 335.754 & 726.871 & 218.347 \\
\hline \multicolumn{5}{|l|}{ Right hemisphere } \\
\hline UF FA & .601 & .045 & .593 & .027 \\
\hline UF RD & .000442 & .0000449 & .000456 & .0000295 \\
\hline UF AD & .00133 & .0000322 & .00134 & .0000254 \\
\hline $\begin{array}{l}\text { UF number of stream- } \\
\text { lines }\end{array}$ & 144.036 & 114.118 & 236.581 & 142.907 \\
\hline IFOF FA & .708 & .027 & .683 & .027 \\
\hline IFOF RD & .000354 & .0000310 & .000386 & .0000312 \\
\hline IFOF AD & .00147 & .0000359 & .00148 & .0000274 \\
\hline $\begin{array}{l}\text { IFOF number of } \\
\text { streamlines }\end{array}$ & 350.255 & 2235.006 & 474.452 & 239.131 \\
\hline CB FA & .609 & .027 & .588 & .029 \\
\hline CB RD & .000421 & .0000304 & .000451 & .0000310 \\
\hline$C B A D$ & .00130 & .0000346 & .00132 & .0000298 \\
\hline $\begin{array}{l}\text { CB number of stream- } \\
\text { lines }\end{array}$ & 995.035 & 341.383 & 818.516 & 283.127 \\
\hline ILF FA & .662 & .024 & .646 & .033 \\
\hline ILF RD & .000396 & .0000307 & .000419 & .0000404 \\
\hline ILF AD & .00140 & .0000588 & .00143 & .0000393 \\
\hline
\end{tabular}

Table 2 continued

\begin{tabular}{|c|c|c|c|c|}
\hline & \multicolumn{2}{|c|}{ 22q11.2DS } & \multicolumn{2}{|l|}{ Control } \\
\hline & $M$ & $S D$ & $M$ & $S D$ \\
\hline $\begin{array}{l}\text { ILF number of stream- } \\
\text { lines }\end{array}$ & 31.684 & 31.783 & 25.065 & 20.855 \\
\hline SLFFA & .649 & .039 & .656 & .043 \\
\hline SLF RD & .000399 & .0000370 & .000401 & .0000491 \\
\hline SLF AD & .00139 & .0000582 & .00142 & .0000417 \\
\hline $\begin{array}{l}\text { SLF number of } \\
\text { streamlines }\end{array}$ & 83.192 & 74.113 & 144.194 & 133.073 \\
\hline Thalamo-frontal FA & .633 & .019 & .621 & .023 \\
\hline Thalamo-frontal RD & .000401 & .0000223 & .000417 & .0000241 \\
\hline Thalamo-frontal AD & .00132 & .0000226 & .00132 & .0000214 \\
\hline $\begin{array}{l}\text { Thalamo-frontal num- } \\
\text { ber of streamlines }\end{array}$ & 821.088 & 318.348 & 769.516 & 239.898 \\
\hline \multicolumn{5}{|c|}{$\begin{array}{l}\text { DTI, diffusion tensor imaging; 22q11.2DS, 22q11.2 deletion syndrome; UF, } \\
\text { uncinate fasciculus; IFOF, inferior fronto-occipital fasciculus; } C B \text {, cingulum } \\
\text { bundle; ILF, inferior longitudinal fasciculus; SLF, superior longitudinal fasciculus; } \\
\text { FA, fractional anisotropy; RD, radial diffusivity; AD, axial diffusivity }\end{array}$} \\
\hline \multicolumn{5}{|c|}{ Bonferroni corrected statistically significant differences indicated in italics } \\
\hline
\end{tabular}

Compared to controls, participants with 22q11.2DS showed a significant increase in the right hemisphere IFOF FA, and a significant decrease in right hemisphere IFOF RD and UF number of streamlines. Figure 1 depicts the reconstructed white matter tracts for which we found significant differences between groups.

\section{Differences in social brain behavioral measures}

To explore group differences in behavior-based social brain measures, we conducted an Analysis of Covariance (ANCOVA) to compare participants with 22q11.2DS and controls for scores on the SRS. We used FSIQ as a covariate to account for the fact that overall intelligence may affect social cognitive ability. Due to the nonnormality of the SRS variable, we applied a log transformation which normalized the SRS data. Descriptive statistics are presented in Table 4. Results indicated significantly higher (i.e., more impaired) scores in the group of individuals with 22q11.2DS (range $=17-140$ ) compared to controls (range $=3-75)[F(1,83)=16.352, p<.0001]$.

We conducted a multivariate analysis of variance (MANOVA) to compare scores between groups on the JSS. Descriptive statistics are presented in Table 4. Results demonstrated no significant differences between groups on the social, cognitive, physical, and perceptual subscales of the JSS; therefore, we did not run an analysis to covary for FSIQ (Table 5). We conducted a nonparametric Mann-Whitney U test for the non-normally distributed JSS Impulsive scale; results also indicated no significant differences between groups $(p=.677)$.

Finally, we conducted a MANCOVA using FSIQ as a covariate to compare scores between groups on the 
Table 3 Results of MANCOVAs for DTI Tracts

\begin{tabular}{|c|c|c|c|c|c|c|}
\hline Tract & Wilks' Lambda & $p$ value & Dependent variable & $F(\mathrm{df})$ & p value & Partial eta squared \\
\hline \multirow[t]{4}{*}{ Left UF } & .899 & .069 & Fractional anisotropy & $.004(1,84)$ & .948 & .000 \\
\hline & & & Radial diffusivity & $.095(1,84)$ & .758 & .001 \\
\hline & & & Axial diffusivity & $2.878(1,84)$ & .093 & .033 \\
\hline & & & Number of streamlines & $.001(1,84)$ & .976 & .000 \\
\hline \multirow[t]{3}{*}{ Right UF } & .758 & $<.0001$ & Fractional anisotropy & $1.308(1,84)$ & .256 & .015 \\
\hline & & & Radial diffusivity & $3.483(1,84)$ & .065 & .040 \\
\hline & & & Axial diffusivity & $4.344(1,84)$ & .040 & .049 \\
\hline \multirow[t]{4}{*}{ Left IFOF } & .678 & $<.0001$ & Fractional anisotropy & $26.542(1,83)$ & $<.0001$ & .242 \\
\hline & & & Radial diffusivity & $34.271(1,83)$ & $<.0001$ & .292 \\
\hline & & & Axial diffusivity & $.762(1,83)$ & .385 & .009 \\
\hline & & & Number of streamlines & $1.581(1,83)$ & .212 & .019 \\
\hline \multirow[t]{2}{*}{ Right IFOF } & 681 & $<.0001$ & Axial diffusivity & $1.457(1,83)$ & .231 & .017 \\
\hline & & & Number of streamlines & $3.111(1,83)$ & .081 & .036 \\
\hline \multirow[t]{4}{*}{ Left CB } & .825 & .003 & Fractional anisotropy & $4.387(1,85)$ & .039 & .049 \\
\hline & & & Radial diffusivity & $8.051(1,85)$ & .006 & .087 \\
\hline & & & Axial diffusivity & $4.091(1,85)$ & .046 & .046 \\
\hline & & & Number of streamlines & $8.681(1,85)$ & .004 & .093 \\
\hline \multirow[t]{4}{*}{ Right CB } & .708 & $<.0001$ & Fractional anisotropy & $14.008(1,85)$ & $<.0001$ & .141 \\
\hline & & & Radial diffusivity & $20.097(1,85)$ & $<.0001$ & .191 \\
\hline & & & Axial diffusivity & $4.739(1,85)$ & .032 & .053 \\
\hline & & & Number of streamlines & $19.858(1,85)$ & $<.0001$ & .189 \\
\hline \multirow[t]{4}{*}{ Left ILF } & .810 & .002 & Fractional anisotropy & $.181(1,85)$ & .671 & .002 \\
\hline & & & Radial diffusivity & $.107(1,85)$ & .744 & .001 \\
\hline & & & Axial diffusivity & $1.352(1,85)$ & .248 & .016 \\
\hline & & & Number of streamlines & $5.167(1,85)$ & .026 & .057 \\
\hline \multirow[t]{4}{*}{ Right ILF } & .803 & .001 & Fractional anisotropy & $7.705(1,85)$ & .007 & .083 \\
\hline & & & Radial diffusivity & $11.332(1,85)$ & .001 & .118 \\
\hline & & & Axial diffusivity & $5.246(1,85)$ & .024 & .058 \\
\hline & & & Number of streamlines & $.571(1,85)$ & .452 & .007 \\
\hline \multirow[t]{4}{*}{ Left SLF } & .885 & .046 & Fractional anisotropy & $.608(1,81)$ & .438 & .007 \\
\hline & & & Radial diffusivity & $.128(1,81)$ & .722 & .002 \\
\hline & & & Axial diffusivity & $.115(1,81)$ & .736 & .001 \\
\hline & & & Number of streamlines & $4.813(1,81)$ & .031 & .056 \\
\hline \multirow[t]{4}{*}{ Right SLF } & .899 & .072 & Fractional anisotropy & $.004(1,83)$ & .952 & .000 \\
\hline & & & Radial diffusivity & $.496(1,83)$ & .483 & .006 \\
\hline & & & Axial diffusivity & $2.787(1,83)$ & .099 & .032 \\
\hline & & & Number of streamlines & $4.252(1,83)$ & .042 & .049 \\
\hline \multirow[t]{4}{*}{ Left thalamo-frontal } & .831 & .004 & Fractional anisotropy & $4.347(1,85)$ & .040 & .081 \\
\hline & & & Radial diffusivity & $7.483(1,85)$ & .008 & .059 \\
\hline & & & Axial diffusivity & $.845(1,85)$ & .361 & .010 \\
\hline & & & Number of streamlines & $1.008(1,85)$ & .318 & .012 \\
\hline \multirow[t]{4}{*}{ Right thalamo-frontal } & .870 & .021 & Fractional anisotropy & $9.116(1,85)$ & .003 & .097 \\
\hline & & & Radial diffusivity & $9.911(1,85)$ & .002 & .104 \\
\hline & & & Axial diffusivity & $.731(1,85)$ & .395 & .009 \\
\hline & & & Number of streamlines & $2.485(1,85)$ & .119 & .028 \\
\hline
\end{tabular}

UF, uncinate fasciculus; IFOF, inferior fronto-occipital fasciculus; CB, cingulum bundle; ILF, inferior longitudinal fasciculus; SLF, superior longitudinal fasciculus Bonferroni corrected statistically significant results indicated in italics $(p<.004)$ 

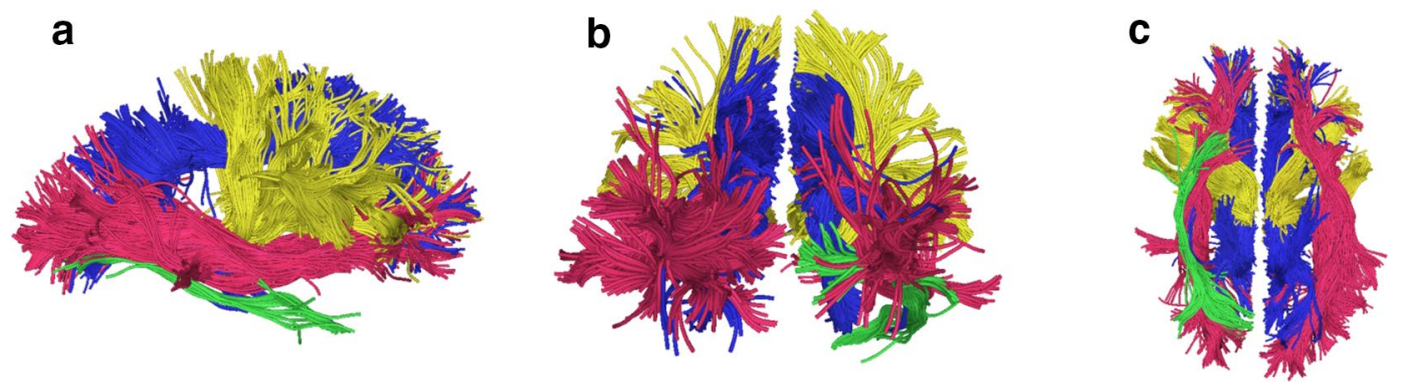

Fig. 1 Fiber tracts of interest. ILF = green, IFOF = red, thalamo-frontal connection = yellow, and CB = blue. a Right lateral view (b) posterior view (c) inferior view

Table 4 Descriptive statistics for social measures

\begin{tabular}{|c|c|c|c|c|}
\hline \multirow[t]{2}{*}{ Measure } & \multicolumn{2}{|c|}{$22 q 11.2 D S$} & \multicolumn{2}{|c|}{ Controls } \\
\hline & $M$ & $S D$ & $M$ & $S D$ \\
\hline SRS total & 73.321 & 3.896 & 18.433 & 5.323 \\
\hline JSS social & 2.930 & 2.412 & 1.700 & 1.841 \\
\hline JSS cognitive & 3.982 & 2.066 & 2.900 & 2.057 \\
\hline JSS perceptual & 1.298 & 1.773 & .533 & 1.047 \\
\hline JSS impulsive & 2.421 & 1.861 & 2.300 & 1.765 \\
\hline JSS physical & 3.579 & 1.927 & 2.600 & 1.632 \\
\hline TEIQue emotion regulation & 3.949 & 1.196 & 5.247 & .841 \\
\hline TElQue empathy & 3.452 & 1.043 & 5.196 & .858 \\
\hline TEIQue social awareness & 3.360 & 1.042 & 5.473 & .965 \\
\hline TEIQue emotion perception & 3.507 & 1.025 & 5.243 & .841 \\
\hline
\end{tabular}

SRS, Social Responsiveness Scale, adult research version; JSS, Junior Schizotypy Scale; TEIQue, Trait Emotional Intelligence Questionnaire; 22q11.2DS, 22q11.2 deletion syndrome

Statistically significant differences indicated in italics

TEIQue. Descriptive statistics are presented in Table 4. Due to nonnormality of the social awareness variable, we ran an ANCOVA using FSIQ as a covariate for the log transformed version of this variable. Results indicated significant differences in scores between individuals with 22q11.2DS and controls $(F[1,83]=13.395, \mathrm{p}<.0001)$.
Therefore, parents/caregivers of individuals with 22q11.2DS rated them significantly lower than parents/ caregivers of controls on three of the four facet scores of interest in this study; emotion regulation did not pass Bonferroni correction (Table 5).

\section{Correlations between DTI tracts and social behavioral measures}

We used Pearson correlations where appropriate and Spearman correlations for variables that remained nonnormal after log transformation to examine the associations between behavioral measures of social processing and the DTI tracts that had significantly differentiated the study groups. Results are displayed in Tables 6 and 7. Among participants with 22q11.2DS, marginally significant Bonferroni-corrected associations were found between the right UF number of streamlines and the JSS Social scale $(\rho=.260, p=.039)$, the right UF number of streamlines and the TEIQue Social Awareness facet $(\rho=-.224, p=.050)$, and the right IFOF RD and JSS Impulsive scale $(\rho=-.380, p=.006)$. We noted significant Bonferroni-corrected positive correlations between the right thalamo-frontal tract RD and TEIQue Empathy facet ( $r=.351, p=.001)$, as well as between the right IFOF FA and the JSS Impulsive scale $(\rho=.412$, $p=.003)$ and the right UF number of streamlines and

Table 5 Results of MANCOVA for social measures

\begin{tabular}{|c|c|c|c|c|c|}
\hline Measure & Wilks' Lambda & p value & Dependent variable & $F(\mathrm{df})$ & $\mathrm{p}$ value \\
\hline \multirow[t]{4}{*}{ Junior Schizotypy Scale } & .934 & .740 & Social & $1.128(1,31)$ & .296 \\
\hline & & & Cognitive & $.003(1,31)$ & .958 \\
\hline & & & Perceptual & $.417(1,31)$ & .523 \\
\hline & & & Physical & $.016(1,31)$ & .901 \\
\hline \multirow[t]{3}{*}{ TElQue } & .801 & .001 & Emotion regulation & $5.667(1,83)$ & .020 \\
\hline & & & Empathy & $14.618(1,83)$ & $<.0001$ \\
\hline & & & Emotion perception & $12.727(1,83)$ & .001 \\
\hline
\end{tabular}

TEIQue, Trait Emotional Intelligence Questionnaire

Bonferroni corrected significant results indicated in italics $(p<.007)$ 


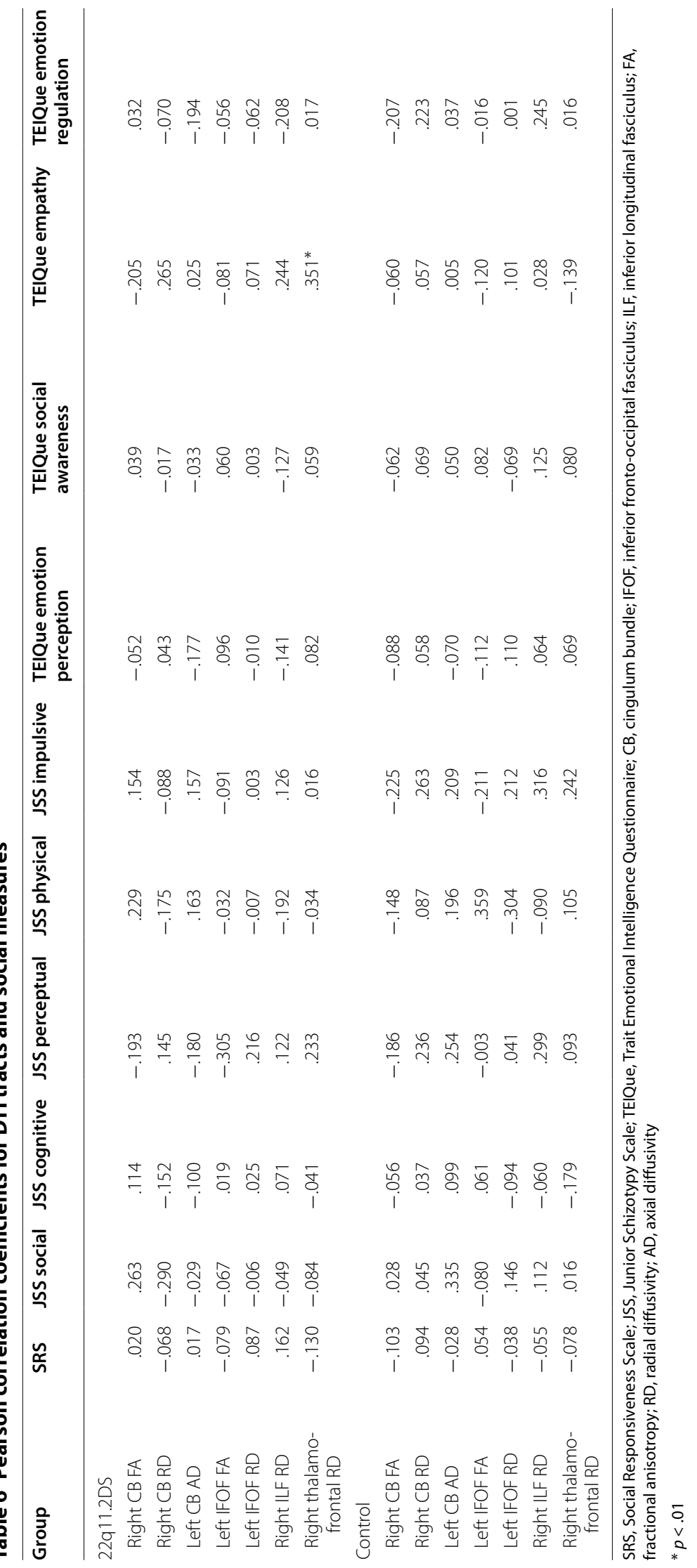




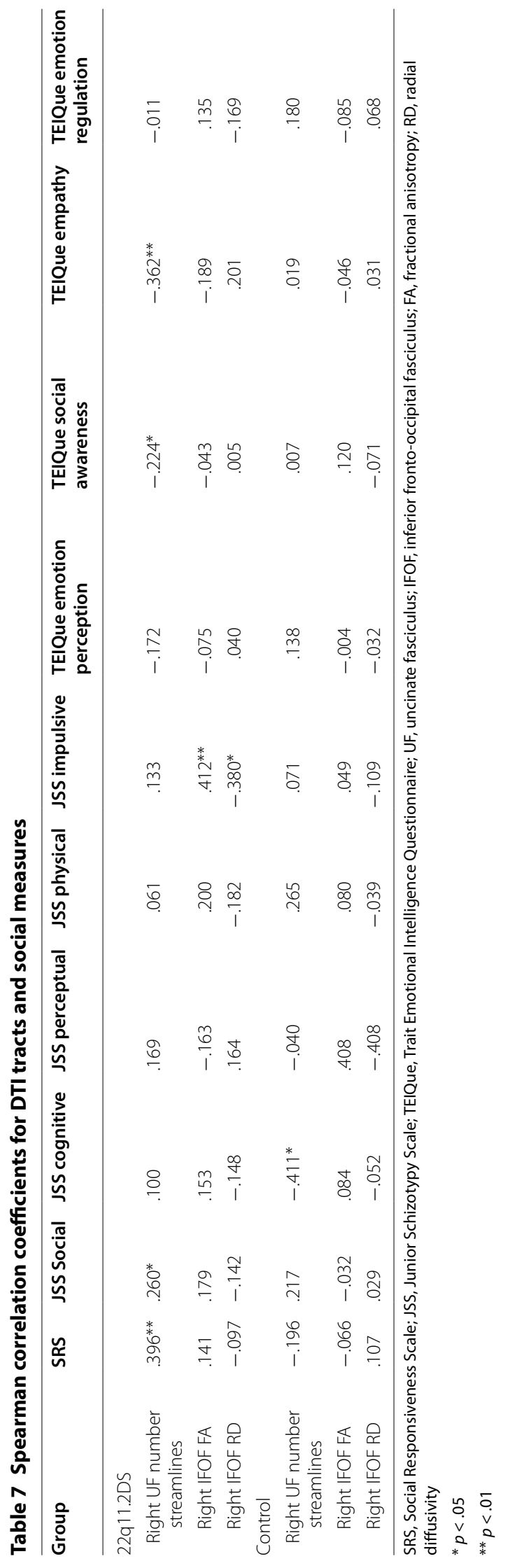


Table 8 Descriptive statistics for 22q11.2DS group by psychosis level

\begin{tabular}{|c|c|c|c|}
\hline Demographic variable & 22q11.2DS no psychosis $(n=43)$ & $22 q 11 . D S+$ prodromal psychosis $(n=10)$ & $22 q 11.2 \mathrm{DS}+$ overt psychosis $(n=4)$ \\
\hline Age [mean (SD)] & 20.65 (2.15) & $22.60(2.50)$ & $19.52(1.76)$ \\
\hline \multicolumn{4}{|l|}{ Gender [N (\%)] } \\
\hline Male & $25(58.1)$ & $5(50.0)$ & $1(25.0)$ \\
\hline Female & $18(41.9)$ & $5(50.0)$ & $3(75.0)$ \\
\hline FSIQ [mean (SD)] & $76.56(12.33)$ & $71.00(6.65)$ & $61.75(5.32)$ \\
\hline
\end{tabular}

22q11.2DS, 22q11.2 deletion syndrome; FSIQ, full scale IQ

Statistically significant differences indicated in italics $(p=.015)$

Table 9 Descriptive Statistics for DTI metrics by psychosis level

\begin{tabular}{|c|c|c|c|c|c|c|}
\hline \multirow[t]{2}{*}{ DTI metric } & \multicolumn{2}{|c|}{ 22q11.2DS no psychosis } & \multicolumn{2}{|c|}{$22 q 11 . D S+$ prodromal psychosis } & \multicolumn{2}{|c|}{ 22q11.2DS + overt psychosis } \\
\hline & $M$ & $S D$ & $M$ & $S D$ & $M$ & $S D$ \\
\hline RH UF number streamlines & 145.488 & 123.879 & 165.000 & 91.459 & 103.750 & 38.638 \\
\hline RHIFOF FA & .702 & .023 & .715 & .016 & .743 & .053 \\
\hline RH IFOF RD & .00036 & .000026 & .00034 & .000023 & .00031 & .000058 \\
\hline $\mathrm{RHCB} F A$ & .609 & .028 & .062 & .027 & .587 & .019 \\
\hline $\mathrm{RH} C \mathrm{CB}$ RD & .00042 & .000031 & .00041 & .000030 & .00043 & .000032 \\
\hline $\mathrm{RH} I L F \mathrm{RD}$ & .00039 & .000028 & .00038 & .000039 & .00039 & .000035 \\
\hline LH IFOF FA & .692 & .025 & .688 & .027 & .679 & .019 \\
\hline LHIFOF RD & .00037 & .000028 & .00037 & .000032 & .00038 & .000026 \\
\hline LHCB AD & .00133 & .000031 & .00133 & .000046 & .00128 & .000012 \\
\hline
\end{tabular}

22q11.2DS, 22q11.2 deletion syndrome; $\mathrm{RH}$, right hemisphere; IFOF, inferior fronto-occipital fasciculus; FA, fractional anisotropy; $R D$, radial diffusivity; $C B$, cingulum bundle; ILF, inferior longitudinal fasciculus; LH, left hemisphere; AD, axial diffusivity

Statistically significant differences identified in italics

Table 10 Results of MANOVA for psychosis level

\begin{tabular}{|c|c|c|c|c|c|}
\hline Group & Wilks' Lambda & p value & DTI Tract & $F(d f)$ & p value \\
\hline \multirow[t]{10}{*}{ Psychosis level } & .459 & .005 & RH UF number streamlines & $.403(2,52)$ & .670 \\
\hline & & & RH IFOF FA & $5.215(2,52)$ & .009 \\
\hline & & & RH IFOF RD & $5.790(2,52)$ & .005 \\
\hline & & & RHCB FA & $1.882(2,52)$ & .162 \\
\hline & & & RH CB RD & $.587(2,52)$ & .560 \\
\hline & & & RHILF RD & $.885(2,52)$ & .419 \\
\hline & & & LHIFOF FA & $.542(2,52)$ & .585 \\
\hline & & & LH IFOF RD & $.040(2,52)$ & .960 \\
\hline & & & LHCB AD & $3.494(2,52)$ & .038 \\
\hline & & & RH thalamo-frontal RD & $1.862(2,52)$ & .166 \\
\hline
\end{tabular}

$\mathrm{RH}$, right hemisphere; UF, uncinate fasciculus; IFOF, inferior fronto-occipital fasciculus; FA, fractional anisotropy; RD, radial diffusivity; CB, cingulum bundle; ILF, inferior longitudinal fasciculus; $\mathrm{LH}$, left hemisphere

the SRS ( $\rho=.396, p=.001$ ). We also found a significant Bonferroni-corrected negative correlation between the right UF number of streamlines and TEIQue Empathy facet $(\rho=-.362, p=.003)$. There were no other significant relationships among the group of individuals with 22q11.2DS.
Among the control group, we found a marginally significant negative correlation after Bonferroni correction between the right UF number of streamlines and JSS Cognitive scale $(\rho=-.411, p=.017)$. There were no other significant correlations between DTI tracts and social brain measures among the control group. 


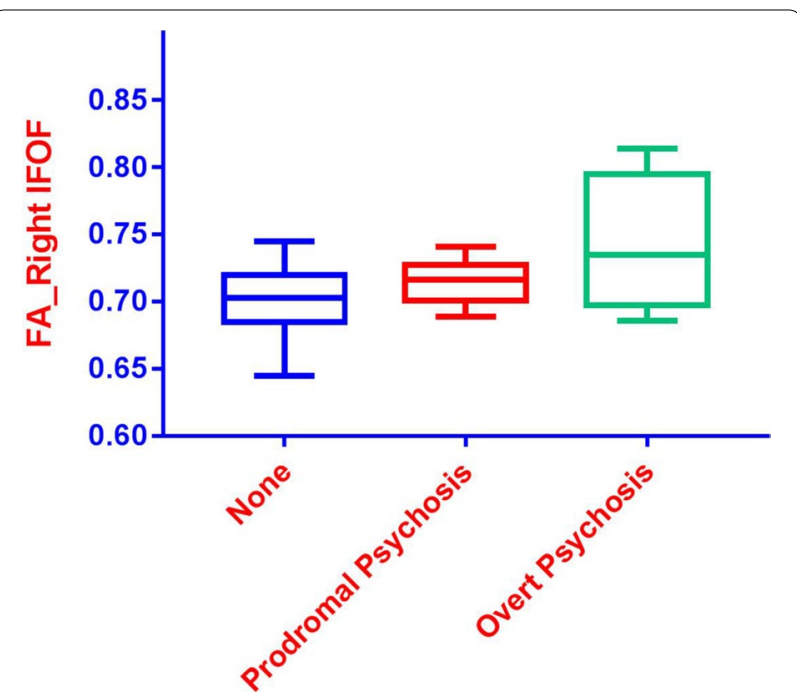

Fig. 2 Boxplot of fractional anisotropy levels for the right IFOF by psychosis level. Blue = no psychosis, Red = prodromal psychosis, Green $=$ overt psychosis

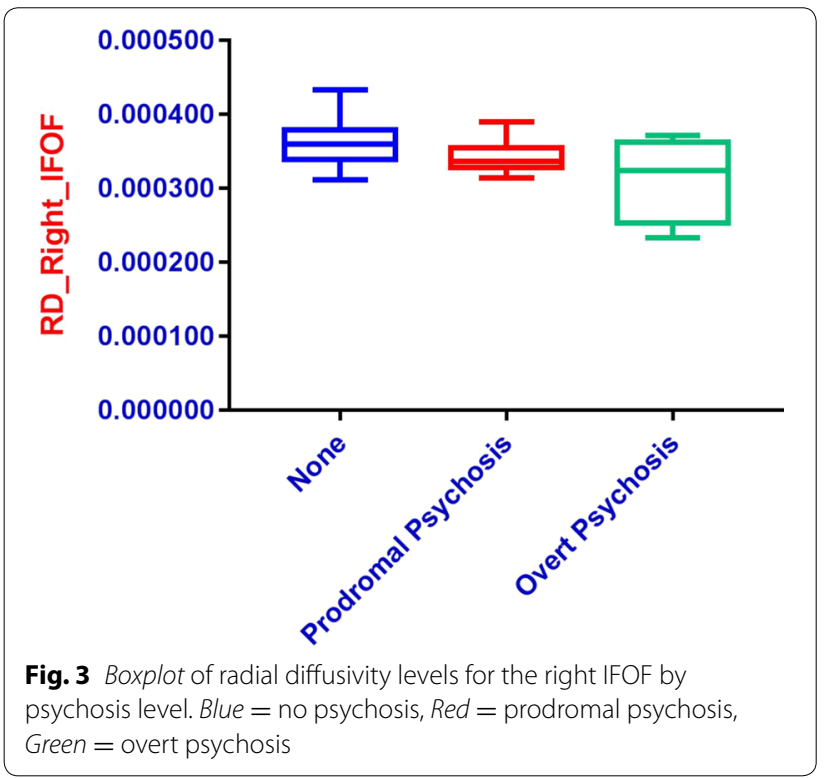

\section{Associations between DTI metrics and symptoms of prodromal/overt psychosis}

In order to examine whether the architecture of white matter tracts affects the development of prodromal symptoms, we ran ZIP regression analyses in order to determine whether there was a relationship between scores on a measure of prodromal symptoms (SIPS PS and SIPS NS) and DTI metrics. Results indicated that, after Bonferroni correction, several DTI metrics were significantly associated with positive symptoms of prodro$\mathrm{mal} /$ overt psychosis, including left IFOF FA $(z=-3.41$, $p=.001)$ and right ILF RD $(z=-4.86, p<.0001)$. DTI metrics that were significantly associated with negative symptoms of prodromal/overt psychosis (after Bonferroni correction) included the right IFOF RD $(z=-8.25$; $p<.0001)$ and the right IFOF FA $(z=7.50, p<.0001)$.

Given these significant results, individuals with 22q11.2DS were further divided into three subgroups: those with no evidence of psychosis (22q11.2DS no psychosis); those with prodromal symptoms (22q11.2DS + prodromal psychosis) based on a score between 3 and 5 on any positive symptom item of the SIPS; and those with overt psychosis (22q11.2DS + overt psychosis) based on a diagnosis of psychotic disorder (Schizophrenia or Psychotic Disorder Not Otherwise Specified) from the SCID. Demographic information is presented in Table 8 . We then conducted an exploratory MANOVA to compare individuals with 22q11.2DS with no psychosis to those with 22q11.2 and prodromal symptoms, and individuals with 22q11.2DS and overt psychosis on the DTI metrics that significantly differentiated individuals with 22q11.2DS from controls. Descriptive statistics for DTI metrics are presented in Table 9. Follow up ANOVAs indicated significant differences in the right hemisphere IFOF FA and RD (Table 10; Figs. 2, 3). Post-hoc Bonferroni-corrected analyses indicated that the group of individuals with 22q11.2DS and overt psychosis showed significant differences in DTI metrics as compared to the other two groups.

\section{Conclusions}

To our knowledge, this is the first study to use twotensor tractography to examine the social brain among individuals with 22q11.2DS. Our results suggest significantly decreased left hemisphere RD in IFOF, and significantly increased FA in the IFOF among individuals with 22q11.2DS. We also found significantly decreased right hemisphere number of streamlines in UF, significantly increased number of streamlines in the $\mathrm{CB}$, significantly decreased RD in IFOF, CB, ILF, and thalamo-frontal tract, while we found significantly increased FA in the IFOF, CB, and thalamo-frontal tract among individuals with 22q11.2DS. In addition, we found significant between group differences on social measures, particularly the SRS and TEIQue. Correlational analyses demonstrated very few associations between DTI tracts and social brain measures; the main significant findings were between the right thalamo-frontal tract RD and TEIQue Empathy facet, the right IFOF FA and the JSS Impulsive scale, the right UF number of streamlines and TEIQue Empathy facet, and the right UF number of streamlines and the SRS. Finally, ZIP regression analyses demonstrated significant associations between the presence of positive prodromal symptoms and left IFOF FA and right 
ILF RD. Negative symptoms were associated with the right IFOF FA and RD metrics.

\section{Alterations in IFOF, CB, and thalamo-frontal tract}

In the present study, we found bilateral increases in FA of the IFOF, and right hemisphere increase in FA for the $\mathrm{CB}$ and thalamo-frontal tract among individuals with 22q11.2DS. This finding is somewhat unexpected, given previous findings of decreased FA in regions of the $C B$ among individuals with schizophrenia [72] and individuals with 22q11.2DS [46, 73], as well as decreased FA in right hemisphere ATR/thalamo-frontal tract in individuals with ASD [53]. Interestingly, a few other studies of individuals with 22q11.2DS have demonstrated findings similar to ours. For example, Jalbrzikowski and colleagues' [74] whole brain analyses found overall increased FA in individuals with 22q11.2DS, regardless of age. The authors attributed this finding to a combination of decreased $A D$ and $R D$, noting that increases in FA have been reported in individuals with other neurodevelopmental disorders [74]. While the cause of increased FA remains unknown, possible suggestions include decreases in axonal branching [75], flattened fibers that enable increased density of white matter [76], or decreased fiber crossing [77]. In a previous set of analyses by our group based on this cohort's assessments at the third timepoint, we found increases in bilateral FA and decreases in bilateral RD in the ALIC, as well as decreases in left hemisphere RD in the UF [45]. We suggested an overall disruption of white matter connectivity as an explanation for these findings, noting that the observed increases in FA in ALIC were driven by the lower RD levels, which in turn suggested changes in myelin development of the ALIC [45]. It is possible that a similar change affected the IFOF and CB in our current set of analyses of Time 4 data.

As stated previously, the IFOF and CB are believed to underlie visual processing/facial emotion recognition and emotional information processing, respectively, whereas the thalamo-frontal/ATR region is believed to play a role in social cognition. These are domains of known difficulty in individuals with 22q11.2DS [39, 40], as well as individuals with ASD [38, 78-80]. Intriguingly, in a study of whole brain tractography in adults with ASD, Roine and colleagues [81] found higher mean FA values for individuals with ASD compared to typically developing controls. The authors suggest the possibility that abnormal synaptic pruning may play a role in the FA increase. They note that strong physical connectivity (e.g., between synapses and tracts) and low computational connectivity (e.g., information transfer) may reinforce each other, adding to the difficulty in differentiating signal from noise [81, 82]. Alternatively, the authors suggest a more strength-based explanation. They note that that social skills and communication training prevalent in the population of individuals with ASD may lead to increased FA values in adults [81]. Research has demonstrated a link between learning a new skill and FA increases [83-85]. Many individuals with 22q11.2DS also participate in social skills or speech therapy/communication training. While these treatments were not examined in the present study, longitudinal studies that take into account the possible impact of social skills training or speech therapy interventions on white matter tracts is an area that warrants further investigation in the 22q11.2DS population.

\section{Impairments in behavioral measures of social processing in 22q11.2DS}

Our results also demonstrated more parent-rated impairments in social processing on the SRS and TEIQue among individuals with 22q11.2DS. These results are similar to those found by other studies. For example, Jalbrzikowski and colleagues [21] found significant impairment among individuals with 22q11.2DS as compared to typically developing controls on a measure of understanding another's intent and on an emotion recognition task [21]. Similarly, Campbell and colleagues [19] found that individuals with 22q11.2DS were less accurate than typically developing controls on measures of emotion identification and attribution [19]. Taken together, these social cognitive findings indicate that early identification of social impairments, particularly emotion identification and recognition, may provide an area for intervention in individuals with 22q11.2DS that could help to prevent or moderate future difficulty with social and adaptive functioning later in life.

\section{Lack of correlations between DTI metrics and measures of social processing}

In contrast to Jalbrzikowski and colleagues [74], we found only a few significant correlations between social brain measures and DTI metrics (right thalamo-frontal tract RD and TEIQue Empathy, right UF number of streamlines and TEIQue Empathy, right UF number of streamlines and SRS, right IFOF FA and JSS Impulsive), whereas a few others were marginally significant. This difference may be related to the different social measures utilized between studies. For example, Jalbrzikowski and colleagues (2014) found that increased AD in the left IFOF and left UF was associated with better scores on the awareness of social inference test (TASIT) in both individuals with 22q11.2DS and controls. In addition, increased $\mathrm{AD}$ in these same regions was also associated with better performance on the Penn Emotion Recognition Test (ER40) in individuals with 22q11.2DS [74]. Both the TASIT and ER40 are computerized measures of 
social processing concepts, as opposed to the parent and self-report measures of social processing utilized in our study. Therefore, our more indirect measures may be less sensitive to social processing abilities.

Differences in our findings may also be related to age differences between the samples. Our sample consisted more of young adult participants (mean age of 22q11.2DS group $=20.87$, range $=17-25$ ), whereas participants in the Jalbrzikowski et al. study were slightly younger and had a wider age range (mean age of 22q11.2DS group $=16.3 \pm 4.3$ ). Given that the developmental trajectory of brain white matter follows a U-shaped curve, with minimum MD and RD/maximum FA levels occurring around 30 years of age [86-88], it is possible that our older sample includes a greater number of individuals who have reached those levels, and may therefore help to explain our different results.

\section{Associations between DTI metrics and symptoms of prodromal/overt psychosis}

The presence of positive prodromal symptoms was related to DTI metrics of increased FA and in the left IFOF, and decreased RD in the right ILF, whereas presence of negative symptoms was associated with increases in FA, and decreases in RD of the IFOF. Taken together with Jalbrzikowski and colleagues [74] findings of a relationship between decreased $\mathrm{AD}$ in bilateral IFOF and increased positive symptom severity, these findings are intriguing, considering the IFOF's role in visual processing/facial emotion recognition and the difficulties with these types of tasks that are seen in individuals with schizophrenia [89]. Although we cannot infer a causal relationship, these findings lend support to the possibility that disrupted axonal coherence in the IFOF may underlie social cognitive impairment and psychotic symptoms in 22q11.2DS [74]. Longitudinal DTI studies could provide further insight as to whether this white matter disruption precedes the development of prodromal symptoms in individuals with 22q11.2DS.

Within the group of individuals with 22q11.2DS, our analysis of psychosis level, while exploratory, shows evidence of a possible biomarker for psychosis in that the right hemisphere IFOF FA was significantly increased, whereas right hemisphere IFOF RD was significantly decreased in individuals with overt psychosis, as compared to those with prodromal symptoms or no psychosis.

\section{Limitations and suggestions}

Our study does include several limitations. As previously noted, this study is a cross-sectional sample of an ongoing longitudinal study. As such, we are unable to draw any causal conclusions regarding the relationships we did find between poor social processing and positive prodromal symptoms. Longitudinal studies that follow the progress of social processing difficulties and the development of prodromal symptoms in 22q11.2DS are needed to help further elucidate this relationship. Secondly, this study did include a relatively small sample size and our groups were unequal, with fewer participants in the control group. While we did find some between group differences, the study may have suffered from reduced statistical power. As a result, differences that may have appeared with a larger sample size may not have been detected. Similarly, our comparisons between individuals with 22q11.2DS and prodromal psychosis and those with overt psychosis also likely suffered from reduced statistical power, and therefore no final conclusions can be drawn from these particular results. Our sample also included some variability, in that approximately $47 \%$ of our participants with 22q11.2DS had either prodromal symptoms of psychosis or other psychiatric diagnoses (as noted in Table 1). While not entirely certain, it is possible that this variability diluted the association between the DTI findings and behavioral measures. While these diagnoses may have affected our findings, they are common in the 22q11.2DS population, and therefore were not used as covariates in statistical analyses. Moreover, the inclusion of siblings in the control sample may have posed a limitation in that siblings of individuals with schizophrenia have been reported to show alterations in social functioning and underlying white matter connectivity, potentially affecting their control status. However, as we note in Additional file 1, sibling controls and community controls did not differ in any social behavioral or DTI measure. In addition, our measures of social processing relied on parent and self-report questionnaires, which may not be particularly sensitive to the construct of interest.

Our study is the first that we know to report on twotensor tractography of white matter tracts in the social brain. More studies using this methodology in individuals with 22q11.2DS are needed to ensure its reliability and validity in this population. Future studies would also benefit from equally sized, larger groups. In addition, studies that combine more direct theory of mind or social cognitive measures with a two-tensor tractography approach may be more sensitive to differences in the social brain network. While quite promising with a small sample size, replication of the results within right hemisphere FA and RD and their relationship to overt psychosis is also needed. Finally, while the current study provides some important findings, longitudinal studies that track white matter development of individuals with 22q11.2DS, particularly the social brain areas of IFOF and ILF, are needed to help identify further possible 
biomarkers for the development of psychotic symptoms in this population.

\section{Additional file}

Additional file 1. These tables depict the results of multiple analyses of variance comparing the mean scores/values between our three study groups, prior to combining the community controls and sibling controls for further analyses. The results of all post-hoc analyses are Bonferroni-corrected.

\section{Abbreviations}

22q11.2DS: chromosome 22q11.2 deletion syndrome; VCFS: velo-cardiofacial syndrome; ADHD: attention-deficit/hyperactivity disorder; ASD: autism spectrum disorder; FA: fractional anisotropy; RD: radial diffusivity; AD: axial diffusivity; CB: cingulum bundle; ALIC: anterior limb of the internal capsule; UF: uncinate fasciculus; SLF: superior longitudinal fasciculus; IFOF: inferior frontooccipital fasciculus; ILF: inferior longitudinal fasciculus; ATR: anterior thalamic region; fMRI: functional magnetic resonance imaging; DTI: diffusion tensor imaging; FISH: fluorescence in situ hybridization; WAIS-III: Wechsler Adult Intelligence Scale, third edition; FSIQ: full scale IQ; VIQ: verbal IQ; PIQ: performance IQ; SRS: Social Responsiveness Scale; JSS: Junior Schizotypy Scale; TEIQue: Trait Emotional Intelligence Questionnaire; SIPS: structured interview for prodromal syndromes; MPRAGE: magnetization prepared rapid acquisition gradient echo; PAT: parallel acquisition techniques; GRAPPA: generalized autocalibrating partially parallel acquisitions; FOV: field of view; DWI: diffusion weighted image; UKF: unscented Kalman filter; SPGR: spoiled gradient recalled echo; FLIRT: FMRIB's linear image registration tool; WMQL: white matter query language; ROI: region of interest; ANOVA: analysis of variance; ANCOVA: analysis of covariance; MANOVA: multivariate analysis of variance; MANCOVA: multivariate analysis of covariance; ZIP: zero-inflated Poisson; TASIT: the awareness of social inference test; ER40: Penn Emotional Recognition Test.

\section{Authors' contributions}

AKO gathered background information, analyzed and interpreted data, and was a major contributor writing the manuscript. ZK, XG, AZ completed two-tensor tractography models and WMQL method. ZK and ILC wrote the imaging part of the methods section. CSG gathered background information. ILC, NM, XG, YR, and AZ were involved in the development of the two-tensor tractography protocol. KMA completed neuropsychological assessment with the participants. KMA and WF completed psychiatric interviews with the participants. MRK, SB, and MES served as consultants for the two-tensor tractography models. WRK designed the study, analyzed and interpreted data. All authors read and approved the final manuscript.

\section{Author details}

${ }^{1}$ Department of Psychiatry, SUNY Upstate Medical University, 750 E. Adams St., Syracuse, NY 13210, USA. ${ }^{2}$ Department of Psychiatry, Brigham and Women's Hospital, Harvard Medical School, Boston, MA, USA. ${ }^{3}$ Syracuse University, Syracuse, NY, USA. ${ }^{4}$ Departments of Psychiatry and Neurology, Massachusetts General Hospital, Harvard Medical School, Boston, MA, USA. ${ }^{5}$ Department of Radiology, Brigham and Women's Hospital, Harvard Medical School, Boston, MA, USA. ${ }^{6}$ VA Boston Healthcare System, Harvard Medical School, Brockton, MA, USA.

\section{Acknowledgements}

Not applicable.

\section{Competing interests}

The authors declare that they have no competing interests.

\section{Availability of data and materials}

The datasets analyzed for the current study are available from the corresponding author upon reasonable request.

\section{Ethics approval and consent to participate}

Ethics approval was obtained from the SUNY Upstate Institutional Review Board. Informed consent was completed with each participant.

\section{Funding}

This work was supported by funding from the National Institutes of Health Grant MH064824 to WRK and MH106793 to ZK

Received: 11 September 2016 Accepted: 5 February 2017

Published online: 16 February 2017

\section{References}

1. Ryan AK, Goodship JA, Wilson DI, Philip N, Levy A, Seidel H, et al. Spectrum of clinical features associated with interstitial chromosome 22q11 deletions: a European collaborative study. J Med Genet. 1997; 34 798-804. http://www.ncbi.nlm.nih.gov/pubmed/9350810.

2. Grati FR, Molina Gomes D, Ferreira JC, Dupont C, Alesi V, Gouas L, et al. Prevalence of recurrent pathogenic microdeletions and microduplications in over 9500 pregnancies. Prenat Diagn. 2015;35:801-9.

3. Shprintzen RJ. Velocardiofacial syndrome. Otolaryngol Clin North Am. 2000;33:1217-40. http://www.ncbi.n/m.nih.gov/pubmed/11449784.

4. Campbell LE, Azuma R, Ambery F, Stevens A, Smith A, Morris $R G$, et al. Executive functions and memory abilities in children with 22q11.2 deletion syndrome. Aust N Z J Psychiatry. 2010. doi:10.3109/00048670903489882.

5. Niklasson L, Gillberg C. The neuropsychology of 22q11 deletion syndrome. A neuropsychiatric study of 100 individuals. Res Dev Disabil. 2010. doi:10.1016/j.ridd.2009.09.001.

6. Woodin M, Wang PP, Aleman D, McDonald-McGinn D, Zackai E, Moss E. Neuropsychological profile of children and adolescents with the 22q11.2 microdeletion. Genet Med. 2001. doi:10.1097/00125817-200101000-00008.

7. Antshel KM, Shprintzen R, Fremont W, Higgins AM, Faraone SV, Kates WR. Cognitive and psychiatric predictors to psychosis in velocardiofacial syndrome: a 3-year follow-up study. J Am Acad Child Adolesc Psychiatry. 2010;49:333-44. http://www.ncbi.nlm.nih.gov/pubmed/20410726.

8. Arnold PD, Siegel-Bartelt J, Cytyrnbaum C, Teshima I, Schachar R. Velocardio-facial syndrome: implications of microdeletion 22q11 for schizophrenia and mood disorders. Am J Med Genet. 2001;105:354-62.

9. Baker KD, Skuse DH. Adolescents and young adults with $22 \mathrm{q} 11$ deletion syndrome: psychopathology in an at-risk group. Br J Psychiatry. 2005. doi:10.1192/bjp.186.2.115.

10. Jolin EM, Weller RA, Weller EB. Occurrence of affective disorders compared to other psychiatric disorders in children and adolescents with 22q11.2 deletion syndrome. J Affect Disord. 2012. doi:10.1016/j.jad.2010.11.025.

11. Antshel KM, Fremont W, Roizen NJ, Shprintzen R, Higgins AM, Dhamoon A, Kates WR. ADHD, major depressive disorder, and simple phobias are prevalent psychiatric conditions in youth with velocardiofacial syndrome. J Am Acad Child Adolesc Psychiatry. 2006. doi:10.1097/01. chi.0000205703.25453.5a.

12. Fabbro A, Rizzi E, Schneider M, Debbane M, Eliez S. Depression and anxiety disorders in children and adolescents with velo-cardio-facial syndrome (VCFS). Eur Child Adolesc Psychiatry. 2012. doi:10.1007/ s00787-012-0273-x.

13. Tang SX, Yi JJ, Calkins ME, Whinna DA, Kohler CG, Souders MC, et al. Psychiatric disorders in 22q11.2 deletion syndrome are prevalent but undertreated. Psychol Med. 2014. doi:10.1017/S0033291713001669.

14. Antshel KM, Aneja A, Strunge L, Peebles J, Fremont WP, Stallone K, et al. Autistic spectrum disorders in velo-cardio facial syndrome (22q11.2 deletion). J Autism Dev Disord. 2007. doi:10.1007/s10803-006-0308-6.

15. Ousley OY, Smearman E, Fernandez-Carriba S, Rockers KA, Coleman K, Walker EF, Cubells JF. Axis I psychiatric diagnoses in adolescents and young adults with 22q11 deletion syndrome. Eur Psychiatry. 2013;28:41722. doi:10.1016/j.eurpsy.2013.06.002

16. Murphy KC, Jones LA, Owen MJ. High rates of schizophrenia in adults with velo-cardio-facial syndrome. Arch Gen Psychiatry. 1999;56:940-5. http://www.ncbi.nlm.nih.gov/pubmed/10530637.

17. Schneider M, Debbane M, Bassett AS, Chow EW, Fung WL, van den Bree $M$, et al. Psychiatric disorders from childhood to adulthood in 22q11.2 deletion syndrome: results from the International Consortium on Brain and Behavior in 22q11.2 Deletion Syndrome. Am J Psychiatry. 2014. doi:10.1176/appi.ajp.2013.13070864 
18. Campbell LE, Stevens AF, McCabe K, Cruickshank L, Morris RG, Murphy DG, Murphy KC. Is theory of mind related to social dysfunction and emotional problems in 22q11.2 deletion syndrome (velo-cardio-facial syndrome)? J Neurodev Disord. 2011;3:152. doi:10.1007/s11689-011-9082-7.

19. Campbell LE, McCabe KL, Melville JL, Strutt PA, Schall U. Social cognition dysfunction in adolescents with 22q11.2 deletion syndrome (velo-cardiofacial syndrome): relationship with executive functioning and social competence/functioning. J Intellect Disabil Res. 2015;59:845. doi:10.1111/ jir.12183.

20. Ho JS, Radoeva PD, Jalbrzikowski M, Chow C, Hopkins J, Tran WC, et al. Deficits in mental state attributions in individuals with 22q11.2 deletion syndrome (velo-cardio-facial syndrome). Autism Res. 2012. doi:10.1002/ aur.1252.

21. Jalbrzikowski M, Carter C, Senturk D, Chow C, Hopkins JM, Green MF, et al. Social cognition in 22q11.2 microdeletion syndrome: relevance to psychosis? Schizophr Res. 2012. doi:10.1016/j.schres.2012.10.007.

22. Green MF, Penn DL, Bentall R, Carpenter WT, Gaebel W, Gur RC, et al. Social cognition in schizophrenia: an NIMH workshop on definitions, assessment, and research opportunities. Schizophr Bull. 2008. doi:10.1093/schbul/sbm145.

23. Pinkham AE, Penn DL, Green MF, Buck B, Healey K, Harvey PD. The social cognition psychometric evaluation study: results of the expert survey and RAND panel. Schizophr Bull. 2014. doi:10.1093/schbul/sbt081.

24. Green MF, Horan WP, Lee J. Social cognition in schizophrenia. Nat Rev Neurosci. 2015. doi:10.1038/nrn4005

25. Senju A. Atypical development of spontaneous social cognition in autism spectrum disorders. Brain Dev. 2013. doi:10.1016/j.braindev.2012.08.002.

26. Lee KH, Farrow TF, Spence SA, Woodruff PW. Social cognition, brain networks and schizophrenia. Psychol Med 2004;34:391-400. http://www. ncbi.nlm.nih.gov/pubmed/15259824.

27. Schlaffke L, Lissek S, Lenz M, Juckel G, Schultz T, Tegenthoff M, et al. Shared and nonshared neural networks of cognitive and affective theoryof-mind: a neuroimaging study using cartoon picture stories. Hum Brain Mapp. 2015. doi:10.1002/hbm.22610.

28. Van Overwalle F, Baetens K, Marien P, Vandekerckhove M. Social cognition and the cerebellum: a meta-analysis of over $350 \mathrm{fMRI}$ studies. Neuroimage. 2014. doi:10.1016/j.neuroimage.2013.09.033.

29. Ferrari PF. The neuroscience of social relations. A comparative-based approach to empathy and to the capacity of evaluating others' action value. Behaviour. 2014. doi:10.1163/1568539X-00003152.

30. lacoboni $M$, Dapretto $M$. The mirror neuron system and the consequences of its dysfunction. Nat Rev Neurosci. 2006. doi:10.1038/nrn2024.

31. Bjorkquist OA, Herbener ES. Social perception in schizophrenia: evidence of temporo-occipital and prefrontal dysfunction. Psychiatry Res. 2013. doi:10.1016/j.pscychresns.2012.12.002.

32. Das P, Lagopoulos J, Coulston CM, Henderson AF, Malhi GS. Mentalizing impairment in schizophrenia: a functional MRI study. Schizophr Res. 2012. doi:10.1016/j.schres.2011.08.019.

33. Dodell-Feder D, Delisi LE, Hooker Cl. The relationship between default mode network connectivity and social functioning in individuals at familial high-risk for schizophrenia. Schizophr Res. 2014. doi:10.1016/j. schres.2014.03.031.

34. Smith MJ, Schroeder MP, Abram SV, Goldman MB, Parrish TB, Wang X, et al. Alterations in brain activation during cognitive empathy are related to social functioning in schizophrenia. Schizophr Bull. 2015. doi:10.1093/ schbul/sbu023.

35. Assaf M, Jagannathan K, Calhoun VD, Miller L, Stevens MC, Sahl R, et al. Abnormal functional connectivity of default mode sub-networks in autism spectrum disorder patients. Neuroimage. 2010. doi:10.1016/j. neuroimage.2010.05.067.

36. Bernhardt BC, Valk SL, Silani G, Bird G, Frith U, Singer T. Selective disruption of sociocognitive structural brain networks in autism and alexithymia. Cereb Cortex. 2014. doi:10.1093/cercor/bht182.

37. Hanson C, Hanson SJ, Ramsey J, Glymour C. Atypical effective connectivity of social brain networks in individuals with autism. Brain Connect. 2013. doi:10.1089/brain.2013.0161

38. Kim SY, Choi US, Park SY, Oh SH, Yoon HW, Koh YJ, et al. Abnormal activation of the social brain network in children with autism spectrum disorder: an FMRI study. Psychiatry Investig. 2015. doi:10.4306/pi.2015.12.1.37.

39. Andersson F, Glaser B, Spiridon M, Debbane M, Vuilleumier P, Eliez S. Impaired activation of face processing networks revealed by functional magnetic resonance imaging in 22q11.2 deletion syndrome. Biol Psychiatry. 2008. doi:10.1016/j.biopsych.2007.02.022.

40. Schreiner MJ, Karlsgodt KH, Uddin LQ, Chow C, Congdon E, Jalbrzikowski M, Bearden CE. Default mode network connectivity and reciprocal social behavior in 22q11.2 deletion syndrome. Soc Cogn Affect Neurosci. 2014. doi:10.1093/scan/nst114.

41. Scariati E, Padula MC, Schaer M, Eliez S. Long-range dysconnectivity in fronal and midline structures is associated to psychosis in 22q11.2 deletion syndrome. J Neural Transm. 2016;123:823-39.

42. Barnea-Goraly N, Menon V, Krasnow B, Ko A, Reiss A, Eliez S. Investigation of white matter structure in velocardiofacial syndrome: a diffusion tensor imaging study. Am J Psychiatry. 2003;160:1863-9. http://www.ncbi.nIm. nih.gov/pubmed/14514502.

43. Kates WR, Olszewski AK, Gnirke MH, Kikinis Z, Nelson J, Antshel KM, et al. White matter microstructural abnormalities of the cingulum bundle in youths with 22q11.2 deletion syndrome: associations with medication, neuropsychological function, and prodromal symptoms of psychosis. Schizophr Res. 2015. doi:10.1016/j.schres.2014.07.010.

44. Kubicki M, McCarley R, Westin CF, Park HJ, Maier S, Kikinis R, et al. A review of diffusion tensor imaging studies in schizophrenia. J Psychiatr Res. 2007. doi:10.1016/j.jpsychires.2005.05.005.

45. Perlstein MD, Chohan MR, Coman IL, Antshel KM, Fremont WP, Gnirke $\mathrm{MH}$, et al. White matter abnormalities in 22q11.2 deletion syndrome: preliminary associations with the Nogo-66 receptor gene and symptoms of psychosis. Schizophr Res. 2014. doi:10.1016/j.schres.2013.11.015.

46. Radoeva PD, Coman IL, Antshel KM, Fremont W, McCarthy CS, Kotkar A, et al. Atlas-based white matter analysis in individuals with velo-cardiofacial syndrome (22q11.2 deletion syndrome) and unaffected siblings. Behav Brain Funct. 2012. doi:10.1186/1744-9081-8-38.

47. Catani M, Thiebaut de Schotten M. A diffusion tensor imaging tractography atlas for virtual in vivo dissections. Cortex. 2008. doi:10.1016/j. cortex.2008.05.004

48. Makris N, Preti MG, Wassermann D, Rathi Y, Papadimitriou GM, Yergatian $C$, et al. Human middle longitudinal fascicle: segregation and behavioralclinical implications of two distinct fiber connections linking temporal pole and superior temporal gyrus with the angular gyrus or superior parietal lobule using multi-tensor tractography. Brain Imaging Behav. 2013. doi:10.1007/s11682-013-9235-2.

49. DeRosse P, Nitzburg GC, Ikuta T, Peters BD, Malhotra AK, Szeszko PR. Evidence from structural and diffusion tensor imaging for frontotemporal deficits in psychometric schizotypy. Schizophr Bull. 2015. doi:10.1093/ schbul/sbu150.

50. Ashtari M. Anatomy and functional role of the inferior longitudinal fasciculus: a search that has just begun. Dev Med Child Neurol. 2012. doi:10.1111/j.1469-8749.2011.04122.x.

51. Ortibus E, Verhoeven J, Sunaert S, Casteels I, de Cock P, Lagae L. Integrity of the inferior longitudinal fasciculus and impaired object recognition in children: a diffusion tensor imaging study. Dev Med Child Neurol. 2012. doi:10.1111/j.1469-8749.2011.04147.x

52. Cho Z, Law M, Chi J, Choi S, Park S, Kammen A, et al. An anatomic review of thalamolimbic fiber tractography: Ultra high resolution direct visualization of thalamolimbic fibers anterior thalamic radiation, superolateral and inferomedial forebrain bundle, and newly identified septum pellucidum tract. Word Neurosurg. 2015;83:54. doi:10.1016/j. wneu.2013.08.22

53. Cheon K, Kim Y, Oh S, Park S, Yoon H, et al. Involvement of the anterior thalamic radiation in boys with high functioning autism spectrum disorders: a diffusion tensor imaging study. Brain Res. 2011. doi:10.1016/j. brainres.2011.08.20

54. Skudlarski P, Jagannathan K, Calhoun VD, Hampson M, Skudlarska BA, Pearlson G. Measuring brain connectivity: diffusion tensor imaging validates resting state temporal correlations. Neuroimage. 2008. doi:10.1016/j.neuroimage.2008.07.063.

55. Teipel SJ, Bokde AL, Meindl T, Amaro E Jr, Soldner J, Reiser MF, et al. White matter microstructure underlying default mode network connectivity in the human brain. Neuroimage. 2010. doi:10.1016/j. neuroimage.2009.10.067.

56. Kates WR, Burnette CP, Bessette BA, Folley BS, Strunge L, Jabs EW, Pearlson GD. Frontal and caudate alterations in velocardiofacial syndrome (deletion at chromosome 22q11.2). J Child Neurol. 2004;19:337-42. http:// www.ncbi.nlm.nih.gov/pubmed/15224707. 
57. Kates WR, Burnette CP, Jabs EW, Rutberg J, Murphy AM, Grados M, et al. Regional cortical white matter reductions in velocardiofacial syndrome: a volumetric MRI analysis. Biol Psychiatry. 2001;49:677-84. http://www.ncbi. nlm.nih.gov/pubmed/11313035.

58. Aneja A, Fremont WP, Antshel KM, Faraone SV, AbdulSabur N, Higgins AM, et al. Manic symptoms and behavioral dysregulation in youth with velocardiofacial syndrome (22q11.2 deletion syndrome). J Child Adolesc Psychopharmacol. 2007. doi:10.1089/cap.2006.0023.

59. Wechsler D. Wechsler adult intelligence scale. 3rd ed. San Antonio: The Psychological Corporation; 1997.

60. Sattler JM. Assessment of children: cognitive applications. 4th ed. La Mesa: Jerome M. Sattler, Publisher, Inc.; 2001.

61. Constantino JN, Gruber CP. Social responsiveness scale (SRS) manual. Los Angeles: Western Psychological Services; 2005.

62. Constantino JN, Davis SA, Todd RD, Schindler MK, Gross MM, Brophy $\mathrm{SL}$, et al. Validation of a brief quantitative measure of autistic traits: comparison of the social responsiveness scale with the autism diagnostic interview-revised. J Autism Dev Disord. 2003;33:427-33. http://www.ncbi. nlm.nih.gov/pubmed/12959421.

63. DiDuca D, Joseph S. Assessing schizotypal traits in 13-18 year olds: revising the JSS. Personality Individ Differ. 1999. doi:10.1016/ S0191-8869(98)00260-8.

64. Petrides KV. Technical manual for the Trait Emotional Intelligence Questionnaires (TEIQue). London: London Psychometric Laboratory; 2009.

65. Miller TJ, McGlashan TH, Rosen JL, Cadenhead K, Cannon T, Ventura J, et al. Prodromal assessment with the structured interview for prodromal syndromes and the scale of prodromal symptoms: predictive validity, interrater reliability, and training to reliability. Schizophr Bull. 2003;29:70315. http://www.ncbi.nlm.nih.gov/pubmed/14989408.

66. Malcolm JG, Michailovich O, Bouix S, Westin CF, Shenton ME, Rathi Y. A filtered approach to neural tractography using the Watson directional function. Med Image Anal. 2010. doi:10.1016/j.media.2009.10.003.

67. Malcolm JG, Shenton ME, Rathi Y. Filtered multitensor tractography. IEEE Trans Med Imaging. 2010. doi:10.1109/TMI.2010.2048121.

68. Fischl B, van der Kouwe A, Destrieux C, Halgren E, Segonne F, Salat DH et al. Automatically parcellating the human cerebral cortex. Cereb Cortex. 2004;14:11-22. http://www.ncbi.n/m.nih.gov/pubmed/14654453.

69. Smith SM, Jenkinson M, Woolrich MW, Beckmann CF, Behrens TE, Johansen-Berg $\mathrm{H}$, et al. Advances in functional and structural MR image analysis and implementation as FSL. Neuroimage. 2004;23(Suppl 1):208-19. doi:10.1016/j.neuroimage.2004.07.051.

70. Wassermann D, Makris N, Rathi Y, Shenton M, Kikinis R, Kubicki M, Westin CF. On describing human white matter anatomy: the white matter query language. Med Image Comput Comput Assist Interv. 2013;16(Pt 1):647-54. http://www.ncbi.nlm.nih.gov/pubmed/24505722.

71. Rathi Y, Kubicki M, Bouix S, Westin CF, Goldstein J, Seidman L, et al. Statistical analysis of fiber bundles using multi-tensor tractography: application to first-episode schizophrenia. Magn Reson Imaging. 2011. doi:10.1016/j. mri.2010.10.005.

72. Whitford TJ, Lee SW, Oh JS, de Luis-Garcia R, Savadjiev P, Alvarado JL, et al. Localized abnormalities in the cingulum bundle in patients with schizophrenia: a diffusion tensor tractography study. Neuroimage Clin. 2014. doi:10.1016/j.nicl.2014.06.003.

73. Sundram F, Campbell LE, Azuma R, Daly E, Bloemen OJ, Barker GJ, et al. White matter microstructure in 22q11 deletion syndrome: a pilot diffusion tensor imaging and voxel-based morphometry study of children and adolescents. J Neurodev Disord. 2010. doi:10.1007/s11689-010-9043-6.

74. Jalbrzikowski M, Villalon-Reina JE, Karlsgodt KH, Senturk D, Chow C, Thompson PM, Bearden CE. Altered white matter microstructure is associated with social cognition and psychotic symptoms in 22q11.2 microdeletion syndrome. Front Behav Neurosci. 2014. doi:10.3389/ fnbeh.2014.00393.
75. Hoeft F, Barnea-Goraly N, Haas BW, Golarai G, Ng D, Mills D, et al. More is not always better: increased fractional anisotropy of superior longitudinal fasciculus associated with poor visuospatial abilities in Williams syndrome. J Neurosci. 2007. doi:10.1523/JNEUROSCI.3591-07.2007.

76. Bode MK, Mattila ML, Kiviniemi V, Rahko J, Moilanen I, Ebeling H, et al. White matter in autism spectrum disorders - evidence of impaired fiber formation. Acta Radiol. 2011. doi:10.1258/ar.2011.110197.

77. Arlinghaus LR, Thornton-Wells TA, Dykens EM, Anderson AW. Alterations in diffusion properties of white matter in Williams syndrome. Magn Reson Imaging. 2011. doi:10.1016/j.mri.2011.07.012.

78. Ameis SH, Catani M. Altered white matter connectivity as a neural substrate for social impairment in Autism Spectrum Disorder. Cortex. 2015. doi:10.1016/j.cortex.2014.10.014

79. Jou RJ, Jackowski AP, Papademetris X, Rajeevan N, Staib LH, Volkmar FR. Diffusion tensor imaging in autism spectrum disorders: preliminary evidence of abnormal neural connectivity. Aust N Z J Psychiatry. 2011. doi :10.3109/00048674.2010.534069

80. Jou RJ, Mateljevic N, Kaiser MD, Sugrue DR, Volkmar FR, Pelphrey KA. Structural neural phenotype of autism: preliminary evidence from a diffusion tensor imaging study using tract-based spatial statistics. AJNR Am J Neuroradiol. 2011. doi:10.3174/ajnr.A2558.

81. Roine U, Roine T, Salmi J, Nieminen-Von Wendt T, Leppamaki S, Rintahaka $P$, et al. Increased coherence of white matter fiber tract organization in adults with Asperger syndrome: a diffusion tensor imaging study. Autism Res. 2013. doi:10.1002/aur.1332.

82. Belmonte MK, Allen G, Beckel-Mitchener A, Boulanger LM, Carper RA, Webb SJ. Autism and abnormal development of brain connectivity. J Neurosci. 2004. doi:10.1523/JNEUROSCI.3340-04.2004.

83. Schlegel AA, Rudelson JJ, Tse PU. White matter structure changes as adults learn a second language. J Cogn Neurosci. 2012. doi:10.1162/ jocn_a_00240.

84. Scholz J, Klein MC, Behrens TE, Johansen-Berg H. Training induces changes in white-matter architecture. Nat Neurosci. 2009. doi:10.1038/ $\mathrm{nn} .2412$.

85. Takeuchi H, Sekiguchi A, Taki Y, Yokoyama S, Yomogida Y, Komuro N, et al. Training of working memory impacts structural connectivity. J Neurosci. 2010. doi:10.1523/JNEUROSCI.4611-09.2010.

86. Cohen AH, Wang R, Wilkinson M, MacDonald P, Lim AR, Takahashi E. Development of human white matter fiber pathways: from newborn to adult ages. Int J Dev Neurosci. 2016. doi:10.1016/j.ijdevneu.2016.02.002.

87. Imperati D, Colcombe S, Kelly C, Di Martino A, Zhou J, Castellanos FX, Milham MP. Differential development of human brain white matter tracts. PLOS ONE. 2011. doi:10.1371/journal.pone.0023437.

88. Westlye LT, Walhovd KB, Dale AM, Bjornerud A, Due-Tonnessen P, Engvig $A$, et al. Life-span changes of the human brain white matter: diffusion tensor imaging (DTI) and volumetry. Cereb Cortex. 2010. doi:10.1093/ cercor/bhp280

89. Kohler CG, Walker JB, Martin EA, Healey KM, Moberg PJ. Facial emotion perception in schizophrenia: a meta-analytic review. Schizophr Bull. 2010. doi:10.1093/schbul/sbn192.

\section{Submit your next manuscript to BioMed Central and we will help you at every step:}

- We accept pre-submission inquiries

- Our selector tool helps you to find the most relevant journal

- We provide round the clock customer support

- Convenient online submission

- Thorough peer review

- Inclusion in PubMed and all major indexing services

- Maximum visibility for your research

Submit your manuscript at www.biomedcentral.com/submit
C BioMed Central 